\title{
Uso do Paradigma de Risco para a Esquistossomose em Áreas Endêmicas no Brasil
}

\author{
The Use of Risk Factor Determination for Schistosomiasis in Endemic \\ Areas in Brazil
}

\author{
Pedro Coura-Filho ${ }^{1}$
}

\begin{abstract}
COURA-FILHO, P. The Use of Risk Factor Determination for Schistosomiasis in Endemic Areas in Brazil. Cad. Saúde Públ., Rio de Faneiro, 10 (4): 464-472, Oct/Dez, 1994.

This study shows the results of risk factor determination for infection with Schistosoma mansoni in endemic areas in Brazil. An association was observed between infection with S. mansoni and a number of general conditions: absence of drinking water in the home, low individual income, illiteracy, and residence in an endemic area for more than five years. In addition to these conditions there was also association with a number of habits (risk factors): agricultural and domestic activities in open water supplies, swimming, and fishing. Analysis of the general conditions that may determine these risk factors indicated that provision of drinking water in the home and access to treatment are basic measures that could be adopted in many cases in order to prevent or control the morbidity of the disease. The efficacy of the use of risk factor determination for indication of control measures for this disease is questioned.
\end{abstract}

Key words: chistosomiasis; Schistosoma mansoni; Risk Factors; Control

\section{INTRODUÇÃO}

A declaração de Alma Ata (1978) propunha como principal meta social dos governos alcançar saúde para todos no ano 2.000. A atenção primária foi colocada como estratégia para alcançar esse objetivo, estabelecendo metas precisas, como cobertura vacinal, fornecimento de água potável, eliminação de excretas e serviços de cuidados primários para todos. Foram adotados critérios de eficiência, eficácia e eqüidade em ações verticalizadas e setoriais, visando à melhoria das condições de vida, principalmente da parte da sociedade que é marginal ao processo de produção e acumulação do capital.

A viabilidade dessa estratégia esbarrou em limites teórico-metodológicos que não deram conta de compreender e articular o processo de produção de doenças com enfoque sistêmico.

${ }^{1}$ Centro de Pesquisas René Rachou da Fundação Oswaldo Cruz. Av. Augusto de Lima, 1.715, Belo Horizonte, MG, 30190-002, Brasil.
Doença, aqui entendida como externalidade de processos coletivos de reprodução da vida.

A crise econômica produzida pelo modelo excludente de acumulação desigual do capital produziu agravamento geral da qualidade de vida e redução dos gastos do "Estado Forte" brasileiro, até então responsável pela atenção à saúde de contingentes populacionais cada vez maiores de miseráveis e carentes excluídos do processo de produção e consumo (Boff, 1993). Como conseqüência, a vida de indivíduos excluídos desse processo é cada vez mais desvalorizada, e a morte evitável passa a fazer parte do cotidiano cada vez mais "normal".

O modelo keynesiano de acumulação de capital adotado no Brasil, culminando no período autoritário, não produziu distribuição de renda. Ao contrário, aumentou as desigualdades entre os que produzem e os que detêm os meios de produção. Uma das conseqüências desse modelo foi o aparecimento de duas parcelas da população: uma que não tem o que comer (32 milhões), e outra que vive a caminho da fome, cujos componentes muitas vezes estão desempregados ou no mercado informal, sem sanea- 
mento, educação, transporte, moradia e assistência médica (40 milhões) (Boff, 1993).

Na epidemiologia, tem sido usado o paradigma descritivo com enfoque unicausal (ambiental) e o explicativo com enfoque multicausal, incluindo variáveis sociais (risco) baseadas em informações rígidas e descontextualizadas do seu processo histórico, obtidas por questionário. $\mathrm{O}$ uso desses paradigmas não deu conta de alterar o processo de produção de doenças no Brasil, e o que se observa é o agravamento do perfil clínico-epidemiológico.

Segundo Barbosa (1984), os paradigmas da determinação ambiental e de risco são parciais, porque elegem doenças por interesse de grupos sociais; são distorcidos, porque escondem problemas de maior relevância; e são perversos, porque não buscam a etiologia das suas determinações no contexto sócio-econômico.

Surge, assim, a necessidade de construção de um novo paradigma na epidemiologia, que seja

"mais amplo e compreensivo, caracterizado por teorias, de causalidade mais sofisticadas, capazes de integrar conceitos sistêmicos e causais interdependentes, pela historicidade da doença e seus determinantes, pela consideração da própria epidemiologia em seu contexto sócio-histórico. (...) A medida da morbidade e a identificação dos fatores de risco correspondentes têm produzido dados frios, úteis somente para um planejamento de saúde burocrático e descontextualizado. Portanto, um conhecimento simbólico e praxiológico é necessário para um tratamento mais concreto das questões.

Metodologicamente, é necessário o desenvolvimento de um programa científico especial para atender a estas demandas, capaz de abordar complexas questões de investigação com estratégias de pesquisa igualmente complexas, combinando técnicas de produção de dados com recursos analíticos de diferentes níveis epistemológicos".

(Almeida-Filho, 1992: 108)

Na construção desse novo paradigma na epidemiologia, é imperativo reconhecer a diversidade determinante no processo de produção do conhecimento, partindo de algumas proprie- dades do real: a) que o processo de determinação em epidemiologia não segue qualquer regra probabilística linear ou logística (Schramm \& Castiel, 1992); b) que é um processo determinado por condições macroestruturais, portanto, historicamente determinado (Smith, 1988); c) seus parâmetros são instáveis, produzindo predições de baixa estabilidade que só permite o uso de parâmetros instáveis modulados pelas próprias regras de instabilidade do processo (Garcia, 1986); d) não possibilita a construção de um padrão, e que, o paradigma de risco não opera o concreto e produz apenas conhecimento virtual (Almeida-Filho, 1992).

\section{USO DO PARADIGMA DE RISCO NA ESQUISTOSSOMOSE NO BRASIL}

É suficiente o conhecimento acumulado sobre a epidemiologia e o controle da esquistossomose. A demanda de prefeituras para desenvolver programas de controle da morbidade e transmissão tem sido cada vez maior, tornando imperativo o planejamento estratégico de medidas de controle, visando a obter maior eficácia com menor custo. É também crescente a preocupação, por parte de epidemiologistas e sanitaristas não comprometidos com a indústria de medicamentos e insumos biotecnológicos de interesse da medicina científica capitalista, no sentido de serem encontradas soluções simples, abrangentes, de baixo custo e maior eficácia na prevenção e controle dessa endemia.

A queda dos indicadores específicos da esquistossomose (prevalência, incidência, intensidade da infecção e de formas clínicas) em área endêmica após tratamento específico é, de fato, observada (Coura-Filho, 1990; Coura-Filho et al., 1992). Porém, é desafio evitar o retorno desses indicadores a níveis anteriores e/ou diminuir de forma duradoura a transmissão da endemia em áreas submetidas a controle e cujas medidas foram interrompidas (Katz et al., 1978; Coura Filho et al., 1994). Para isso, é necessário alterar a dinâmica de transmissão.

O paradigma de risco na epidemiologia tem contribuído para o entendimento da associação de variáveis a uma dada doença (Bachet et al., 1985; Almeida-Filho, 1992; Cesar, 1990; Rego et al., 1990). A identificação dos fatores de 
risco para doenças socio-dependentes, como, por exemplo, as infectocontagiosas de veiculação hídrica e as crônicas não-transmissíveis, é possível por meio da análise multivariada com a qual a regressão logística opera (Campos-Filho \& Franco, 1989).

O uso do paradigma de risco para a infecção pelo $S$. mansoni em áreas endêmicas brasileiras tenta explicar a transmissão dessa endemia em nível local, sem levar em consideração a etiologia dos determinantes macroestruturais envolvidos na produção do processo de transmissão. Qual é, então, a real capacidade de contribuição do paradigma de risco para a alteração das condições locais e ambientais que venham diminuir a transmissão?

Neste estudo procurou-se avaliar o uso do paradigma de risco em sete áreas endêmicas para a esquistossomose no Brasil, visando a colaborar na construção de um modelo conceitual dos fatores causais envolvidos na dinâmica de transmissão da endemia.

Nessas áreas, foram identificadas condições ambientais associadas à infecção esquistossomótica (fatores de risco) sem considerar sua etiologia no processo de acumulação desigual do capital, que, em última instância, define a qualidade de vida. Observou-se ainda que cada área apresentou associação diferente de fatores que, conjugados, produziram risco de infecção (Tabela 1).

As condições gerais associadas à transmissão da endemia nas áreas estudadas foram: o fato de a população não ter acesso ao tratamento específico, em três das quatro $(75,0 \%)$ áreas analisadas; ausência de água potável intra-domiciliar, em três de seis $(50,0 \%)$; a população ter migrado de área endêmica onde morou por mais de cinco anos, em três de oito (27,5\%); analfabetismo, em duas de oito $(25,0 \%)$; e renda inferior a meio salário mínimo, em um de oito analisadas (12,5\%).

Os fatores de risco foram: pescar, em seis de oito áreas analisadas (75,0\%); exercer atividades agrícolas, em três de sete (42,9\%); nadar em água a céu aberto, em três de oito (37,5\%); e exercer atividades domésticas, em duas de sete áreas analisadas (29,5\%) (Tabela 1). Assim, a análise multivariada de fatores determinantes na transmissão da esquistossomose apontou as atividades profissionais (agrícolas e domésticas) e as de lazer (pescar e nadar) como sendo os hábitos associados à transmissão da endemia mais freqüente nessas áreas.

As condições gerais identificadas como as possíveis de alterar a probabilidade de infecção a curto e médio prazo foram: ausência de acesso ao tratamento específico e fornecimento de água potável intradomiciliar (Tabela 2).

\section{DISCUSSÃO E CONCLUSÕES}

Um estudo realizado por Barreto (1993) sobre levantamento, por questionário, de informações sobre contato de indivíduos com águas naturais em área endêmica de esquistossomose evidenciou que, com um pequeno número de variáveis, é possível obter informações com alto valor preditivo positivo para identificar indivíduos infectados pelo $S$. mansoni; essa técnica apresenta, além disso, baixo custo operacional.

Neste estudo chegamos a resultados semelhantes, identificando um pequeno número de fatores de risco associados à prevalência da esquistossomose: atividades profissionais (agrícola e doméstica) e de lazer (pescar e nadar). Das condições gerais, as mais freqüentes foram a falta de oportunidade de tratamento específico e a falta de água potável intradomiciliar.

Anterior ao uso do paradigma de risco, o efeito de medidas de controle para a esquistossomose foi simulado por modelos matemáticos, estudados teoricamente por MacDonald (1965), Nasell \& Hirsch (1973), May (1977) e analisados por Woolhouse (1991). Há consenso entre esses autores sobre o fato de tanto o tratamento em massa quanto o seletivo reduzir a prevalência da esquistossomose. Os modelos matemáticos propostos por MacDonald (1965), Goddard (1978), Anderson \& Medley (1985) e Dietz \& Renner (1985) indicam queda abrupta da prevalência após tratamento. Já os propostos por Rosenfield et al. (1977) e Wiemer (1989) indicam também queda da prevalência, porém independente do nível da infecção anterior ao tratamento, o que é considerado irrealista por Woolhouse (1991).

Vários estudos realizados em áreas endêmicas para $S$. mansoni submetidas a medidas de controle confirmam os modelos de Rosenfield et al. e de Wiemer, embora ocorram exceções 


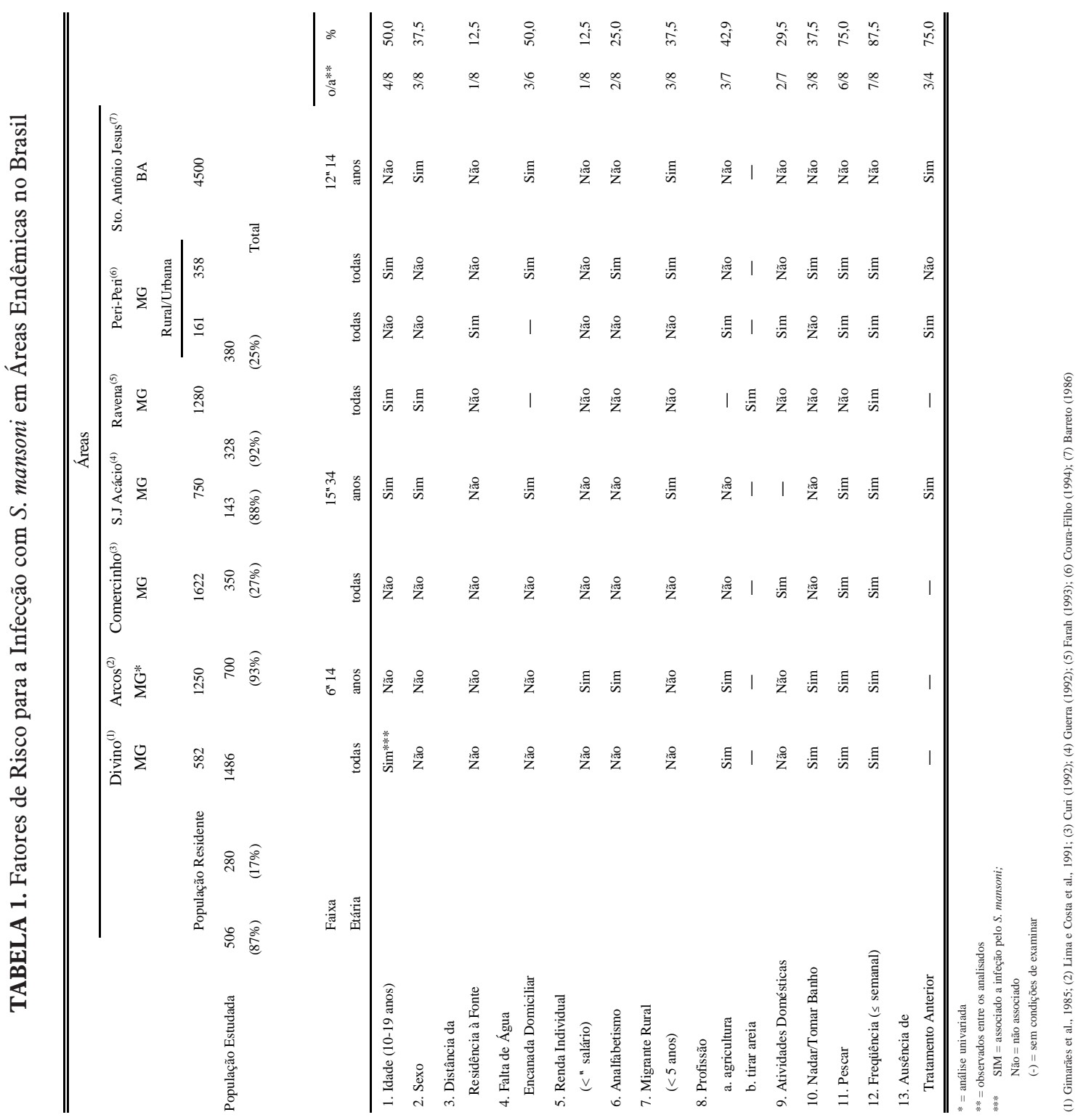


TABELA 2. Condições Gerais e Fatores de Risco para a Infecção pelo S. mansoni no Brasil

\begin{tabular}{|c|c|c|c|c|}
\hline \multicolumn{2}{|c|}{ Condições Gerais } & \multicolumn{2}{|c|}{ Fatores de Risco } & \multirow{2}{*}{$\begin{array}{c}\text { Proporção } \\
\text { do Tempo } \\
\text { de Exposição** }\end{array}$} \\
\hline Variáveis & $\% *$ & Variáveis & $\%$ & \\
\hline Sem Opções de Tratamento & 75,0 & Lazer & & $62-70 \%$ \\
\hline Falta de Água intradomiciliar & 50,0 & $\begin{array}{l}\text { " pescar } \\
\text { " nadar }\end{array}$ & $\begin{array}{l}75,0 \\
37,5\end{array}$ & \\
\hline Analfabetismo & 25,0 & & & \\
\hline Residência em Área Endêmica & 125 & $\begin{array}{l}\text { Atividades } \\
\text { Profissionais }\end{array}$ & & $0-38 \%$ \\
\hline & & " agricultura & 42,9 & \\
\hline Baixa Renda (<" salário) & 12,5 & "doméstica & 29,5 & \\
\hline
\end{tabular}

* Percentagem de áreas estudadas onde ocorreu o fator de risco.

* $\quad$ Proporção do tempo de exposição dos indivíduos a águas naturais em áreas estudadas por Farooq \& Mallah (1966), 67,3\%; Dalton (1976), 66,0\%; Lipes \& Hiati (1977), 70,0\% e Coura-Filho et al., (1985), 62,0\%.

quando há grande fluxo migratório da população (Kloetzel, 1959) ou grande agregação entre indivíduos expostos (Anderson \& Medley, 1985).

O fornecimento de água potável foi considerado por Nasell \& Hirsch (1973) e May (1977) a medida mais efetiva e duradoura na prevenção da infecção esquistossomótica, mesmo quando comparado com a presença de fossas.

Uma revisão sobre o impacto do suprimento de água potável na transmissão da esquistossomose sugere que: "using sophisticated computer modeling techniques it has been predicted that partial redution of water contact should have a proportionately greater effect in diminishing transmission than would similar reductions in snail populations" (Hunter et al., 1993: 13). Nessa revisão, referente a dados de 18 estudos, estimou-se que ocorre redução de $20 \%$ dos contatos dos residentes de área endêmica com águas naturais após fornecimento de água potável. Mas a redução pode ser lenta. Observou-se, em Peri-Peri (Capim Branco, Minas Gerais, Brasil), que $40 \%$ das donas de casa continuavam lavando roupas em córregos e rios após fornecimento de água potável intradomiciliar, devido ao prazer de exercer essa atividade em maior volume de água. Mesmo após seis anos, $20 \%$ das donas-de-casa que tinham água intradomiciliar continuavam lavando roupas em águas naturais (Coura-Filho et al., 1985).

Teoricamente, o fornecimento de água potável intradomiciliar diminui a transmissão da esquistossomose em áreas onde estão presentes a necessidade de tomar banho higiênico e as atividades domésticas em águas infestadas com cercária. Entretanto, exercer atividades agrícolas, tirar areia, nadar e pescar não a afetariam, visto que são, na maioria das vezes, práticas realizadas fora do domicílio e têm, na sua determinação, a necessidade imposta pelo trabalho e/ou lazer.

Nadar e pescar, muitas vezes, têm em comum o lazer que, em muitos casos, ocorre no mesmo tempo e lugar, fazendo parte de um mesmo processo. Embora, em algumas áreas, nadar e pescar possam ser hábitos responsáveis por baixos valores de risco atribuível populacional (Coura-Filho et al., 1994), eles podem chegar a representar até $95,5 \%$ do tempo de exposição de indivíduos em cursos d'água (Dalton, 1976). Além do tempo de exposição nessas atividades, em estudos realizados por Farooq \& Mallah (1966), Dalton (1976), Lipes \& Hiati (1977) e Coura-Filho et al., (1985), o lazer foi motivo de contato para 67,$3 ; 66,0$; 70,0 e 62,0\% dos residentes das áreas por eles estudadas, respectivamente. Ou seja, embora esses hábitos possam ocorrer em pequena parcela da população, a intensidade da expo- 
sição é quase sempre alta nesses casos, levando ao maior risco de indivíduos infectados evoluírem para a forma grave da endemia. Como se percebe, o paradigma de risco não apreende aspectos de processos complexos de transmissão no nível local, além de ofuscar a etiologia dos fatores de risco no nivel macroestrutural.

Certamente, fornecer tratamento específico aos infectados e água potável intradomiciliar são medidas preconizadas muito antes do uso do paradigma de risco para a infecção esquistossomótica. Portanto, seu papel tem sido apenas o de referendar de forma sofisticada aquilo de que a observação direta já havia dado conta e, principalmente, o de explicar a transmissão persistente após implementação de medidas de controle dessa endemia.

A produção de conhecimentos fornecidos pelo uso, até então praticado na epidemiologia dos paradigmas, ambiental e de risco, deve ser somada à necessidade de formulação de um paradigma de ação que possibilite alterar processos focais de produção de doenças. No caso da esquistossomose, têm sido propostas táticas alternativas que levem em consideração a organização local dos serviços de saúde, incluindo o controle da esquistossomose nas ações primárias conforme a realidade local (Barbosa \& Coimbra Jr., 1992).

Nesse tipo de formulação, é imperativo que se inclua o indivíduo, visto que é, ao mesmo tempo, vítima e elemento ativo na trasmissão dessa endemia. A inclusão do individual como parte que contém o todo abre uma perspectiva nova de intervenção no cotidiano. Essa inclusão pressupõe a presença do indivíduo como possibilidade de perceber-se enquanto parte promotora e vítima do processo saúde/doença (S/D). A perspectiva de o indivíduo tornar-se sujeito e objeto do seu processo de vida é uma estratégia de sobrevivência de uma parcela da população que convive com crescentes riscos de adoecer e morrer.

A "epidemiologia comunitária" (Tognoni, 1993), a "pesquisa participante” (Borda, 1981), a "educação popular" (Valla et al., 1993) e a sugestão de um "modelo alternativo", visando ao controle da esquistossomose (Barbosa \& Coimbra Jr., 1992), aparecem como estratégias de apreensão do indivíduo na sua globalidade, de forma a trazer seu universo simbólico-cul- tural, sua instrução histórica e sua "visão de mundo" e do processo S/D para instrumentalizar a elaboração de um paradigma de ação que dê conta de reduzir processos locais de produção de doenças.

Mas, para alguns, essas estratégias apresentam limites metodológicos considerados ideológicos. A "objetividade" da análise quantitativa também o é, na medida em que define $a$ priori seus objetivos, sua metodologia, parâmetros de avaliação e até mesmo resultados previsíveis. Outro limite apontado em "transformar o pesquisado em pesquisador” é a sofisticação teórica e metodológica das ciências que são acessíveis somente aos "iniciados".

Embora os resultados obtidos por esses modelos sejam tópicos, não generalizáveis, com validade somente em nível local, dificultando a produção de conhecimentos científicos que venham alterar as condições estruturais de vida de forma duradoura; essas são estratégias a serem experimentadas na prática, visando a colaborar na construção de um paradigma de ação centrada no indivíduo em seu cotidiano, a ser testado dentro da proposta da descentralização dos serviços de saúde, já adotada pelo Sistema Único de Saúde.

Mas, de fato, a viabilidade das considerações aqui formuladas é desafio para operadores sanitários, planejadores, epidemiologistas e classes subalternas participarem na construção de um paradigma de ação que possa colaborar em desmontes de processos locais de produção de doenças. No caso da esquistossomose, que já se tem conhecimento acumulado suficiente sobre sua epidemiologia de forma a permitir seu controle, esta decisão ética (Guattari, 1992) é urgente sob pena desta endemia se tornar apenas mais uma doença eleita para produzir mais valia do capital através de biotecnologia de diagnóstico e produção de insumos que legitimam a medicina científica.

\section{AGRADECIMENTOS}

$\mathrm{Na}$ elaboração desta revisão contamos com as valiosas sugestões do Prof. Frederico Simões Barbosa e do Dr. Naftale Katz a quem muito agradecemos. 


\section{RESUMO}

\section{COURA-FILHO, P. Uso do Paradigma de} Risco para a Esquistossomose em Áreas Endêmicas no Brasil. Cad. Saúde Públ., Rio de Janeiro, 10 (4): 464-472, out/dez, 1994.

Neste estudo são confrontados os resultados do uso do paradigma de risco na infecção pelo Schistosoma mansoni em áreas endêmicas no Brasil. Foi observada associação da infecção pelo $S$. mansoni com algumas condições gerais: ausência de água potável intradomiciliar, baixa renda individual, analfabetismo e residência atual ou anterior em área endêmica por mais de cinco anos. Além dessas condições ocorreu associação com alguns hábitos (fatores de risco) exercer atividades agrícolas e domésticas em águas a céu aberto, nadar e pescar. A análise das condições gerais que podem estar determinando os fatores de risco, apontou o fornecimento de água potável intradomiciliar e o acesso ao tratamento como medidas abrangentes que muitas vezes podem ser adotadas, visando à prevenção e/ou controle da morbidade da endemia. É questionada a eficácia do uso do paradigma de risco na indicação de medidas de controle dessa endemia.

Palavras-Chave: Esquistossomose; Schistosoma mansoni; Fatores de Risco; Controle

\section{REFERÊNCIAS BIBLIOGRÁFICAS}

AlmA ATA, 1978. Cuidados Primários em Saúde. Genebra: WHO/Unicef.

ALMEIDA-FILHO, N., 1992. A Clínica e a Epidemiologia. Salvador: APCE/Rio de Janeiro: Abrasco.

ANDERSON, R. M. \& MEDLEY, G. F., 1985. Schistosome economics. Parasitology Today, 3: 350-351.

BACHETT, E. M.; DAVIES, A. M. \& PETROSBARVAZIAN, A., 1985. O Enfoque de Risco na Assistência à Saúde: com Especial Referência à Saúde Materno-Infantil, inclusive Planejamento Familiar. Washington, D.C.: Opas. (Publicação Científica, 491)
BARBOSA, F. S., 1984. A epidemiologia como instrumento de análise. Senecta, 7: 31-35.

BARBOSA, F. S. \& COIMBRA Jr., C. E. A., 1992. Alternative approaches in Schistosomiasis control. Memórias do Instituto Oswaldo Cruz, (IV): $215-220$.

BARRETO, L. C., 1986. Epidemiology of Urban Schistosomiasis mansoni in Northeast Brazil. A Case Study in Santo Antonio de Fesus. Tese de Doutorado, London: University of London.

BARRETO, M. L., 1993. Use of risk factors obtained by questionaires in the screening for Schistosoma mansoni infection. American fournal of Tropical Medicine and Hygiene, 48: 742-747.

BOFF, W., 1993. Elementos para uma Pedagogia do Excluído. Petrópolis. (Mimeo.)

BORDA, O. F., 1981. Aspectos teóricos da pesquisa participante. In: Repensando a Pesquisa Participante (C. R. Brandão, org.), pp. 42-62, São Paulo: Brasiliense.

CAMPOS-FILHO, N. B. S. \& FRANCO, E. F., 1989. A microcomputer program for multiple logistic regression by unconditional and conditional maximum likelihoods. American fournal of Epidemiology, 129: 439-344.

CESAR, C. L. G., 1990. Fatores de risco associados à mortalidade infantil em duas áreas da região metropolitana de São Paulo (Brasil), 1984-85. Proposta de instrumentos preditivos. Revista de Saúde Pública, 24: 300-310.

COURA-FILHO, P., 1990. Avaliação de um programa municipalizado de controle da esquistossomose mansoni em Peri-Peri (Capim Branco) MG (Brasil). Tese de Mestrado, Belo Horizonte: Universidade Federal de Minas Gerais.

COURA-FILHO, P; ROCHA, R. S. \& KATZ, N., 1985. Estudo do Contato com Águas Naturais em uma População de Área Endêmica de Esquistossomose em Minas Gerais. Congresso da Sociedade Brasileira de Parasitologia, Fortaleza, CE. (Mimeo.)

COURA-FILHO, P.; ROCHA, R. S.; LIMA E COSTA, M. F. F. \& KATZ, N., 1992. A municipal level approach to the management of schistosomiasis control in Peri-Peri, MG - Brazil. Revista do Instituto de Medicina Tropical de São Paulo, 34: 543-548.

COURA-FILHO, P.; ROCHA, R. S.; SILVA, G. C.; FARAH, M. W. \& KATZ, N., 1994. Identification of risk and groups at risk of infection with Schistosoma mansoni: a estrategy for the implementation of control measures? Revista do Instituto de Medicina Tropical de São Paulo, 36: 245-253. 
CURI, G. C., 1992. Estudo Clínico-Epidemiológico da Esquistossomose mansoni em Comercinho, Minas Gerais (1974, 1981 e 1988). Tese de Doutorado, Belo Horizonte: Universidade Federal de Minas Gerais.

DALTON, G. R., 1976. A socioecological approach to the control of Schistosoma mansoni in St. Lucia. Bulletin of the World Health Organization, 54: 587-595.

DIETZ, K. \& RENNER, H., 1985. A simulation model for the control of helminth diseases by chemoterapy. In: Lecture Notes en Biomathematics (V. Capasso, E. Grosso \& S. L. Paveri-Fontana, eds.), vol. 57, pp. 115-125, Berlin: Springer-Verlag.

FARAH, M. W., 1993. Determinantes Epidemiológicos da Esquistossomose em Ravena, MG. Tese de Mestrado, Belo Horizonte: Universidade Federal de Minas Gerais.

FAROOQ, M. \& MALLAH, M..B., 1966. The behavioural pattern or social and religious projects area. Bulletin of the World Health Organization, 35: 377.

GARCIA, B. R., 1986. Conceptos basicos para el estudio de sistemas complexos. In: Los Problemas del Conocimiento y la Perspectiva Ambiental del Desarollo (E. Leff, org.), pp. 45-71, Mexico: Siglo XXI.

GODDARD, M. J., 1978. On Macdonald's model for schistosomiasis. Transanctions and Royal Tropical Medicine and Hygiene, 72: 123-131.

GUATTARI, F., 1992. Caosmose - um Novo Paradigma Estético. Rio de Janeiro: Ed. 34.

GUERRA, H., 1992. Epidemiologia da Esquistossomose mansoni em São fosé do Acácio, MG. Análise Multivariada dos Fatores Associados à Infecção. Variáveis Sócio-Demográficas e Contatos com Águas Naturais. Tese de Mestrado, Belo Horizonte: Universidade Federal de Minas Gerais.

GUIMARÃES, M. D. C.; LIMA E COSTA, M. F. F; LIMA, L. B. \& MOREIRA, M. A., 1985. Estudo clínico-epidemiológico da esquistossomose mansoni em escolares da Ilha, Município de Arcos, MG (Brasil). Revista de Saúde Pública, 19: 08-17.

HUNTER, J. M.; REY, L.; CHU, K. Y.; ADEKOLU-JHON, E. O. \& MOTT, K. E., 1993. Parasit Diseases in Water Resources Development - The Meed for Intersectorial Negotiation. Geneva: WHO.

KATZ, N.; ZICKER, F.; ROCHA, R. S. \& OLIVEIRA, V. B., 1978. Reinfection of patientes in schistosomiasis mansoni endemic areas after specific treatment. I. Influence of age and worn burden. Revista do Instituto de Medicina Tropical de São Paulo, 20: 273-314.

KLOETZEL, K., 1959. Algumas observações da epidemiologia da esquistossomose na infância. $O$ Hospital, 55: 35-43.

LIMA E COSTA, M. F. F.; ROCHA, R. S.; LEITE, M. L. C.; CARNEIRO, R. G.; COLLEY, D.; GAZZINELLI, G. \& KATZ, N., 1991. A multivariate analysis of socio-demographic factors, water contact patterns and Schistosoma mansoni infection in an endemic area in Brazil. Revista do Instituto de Medicina Tropical de São Paulo, 33: 58-63.

LIPES, J. K. \& HIATI, R. A., 1977. Determinants of humam water contact patters in urban Puerto Rico with special reference to Schistosomiasis. Boletin da Associação Médica de Porto Rico, 69: 35-44.

MacDONALD, G., 1965. The dinamics of helminth infections, with special reference to schistosomes. Transactions Royal Society Tropical Medicine and Hygiene, 59: 489-506.

MAY, R. M., 1977. Togetherness among schistosomes: its effects on the dynamics of the infection. Mathematical Biosciences, (35): 301-343.

NASELL, I. \& HIRSCH, W. M., 1973. The transmission dynamics of schistosomiasis. Community Pure Application Mathematical, 26: 395-453.

REGO, R. A.; BERARDO, F. A. N.; RODRIGUES, S. S. R.; OLIVEIRA, Z. M. A.; OLIVEIRA, M. B.; VASCONCELOS, C.; AVENTURADO, L. V. O.; MONCAU, J. E. C. \& RAMOS, L. R., 1990. Fatores de risco para doenças crônicas não-transmissíveis: inquérito domiciliar no município de São Paulo, SP (Brasil). Metodologia e resultados preliminares. Revista de Saúde Pública, 24: 277-285.

ROSENFIELD, P. L.; SMITH, R. A. \& WOLMAN, M. G., 1977. Development and verification of a schistosomiasis transmission model. American Fournal of Tropical Medicine and Hygiene, 26: 505-516.

SCHRAMM, F. R. \& CASTIEL, L. D., 1992. Processo saúde/doença e complexidade em epidemiologia. Cadernos de Saúde Pública, 8: 379390.

SMITH, N., 1988. Desenvolvimento Desigual: Natureza, Capital e a Produção do Espaço. Rio de Janeiro: Bertrand Brasil.

TOGNONI, G., 1993. Epidemiologia comunitaria. Apuntes para una nueva epidemiologia. Saludy Sociedad, 20: 04-10. 
VALLA, V. V.; CARVALHO, M. \& ASSIS, M., 1993. Participação Popular e os Serviços de Saúde: o Controle Social como Exercício de Cidadania. Rio de Janeiro: Abrasco/Pares.

WIEMER, C., 1989. Optimal disease control enrough combined use of preventive and curative measures. Fournal of Development and Economy, 25: 301-319.
WOOLHOUSE, M. E. J., 1991. On the application of mathematical models of schistosome transmission dynamics. I. Natural transmission. Acta Tropica, 49: 241-270. 